\title{
Discrete Morse Functions from Fourier Transforms
}

\author{
Alexander Engström
}

\section{CONTENTS}

1. Introduction

2. Discrete Morse Theory

3. Fourier Transforms

4. Constructing Discrete Morse Functions

Acknowledgments

References
2000 AMS Subject Classification: Primary 57Q99; Secondary 57R70, 42B10

Keywords: Discrete Morse theory, Fourier transforms, simplicial complexes, Boolean functions
A discrete Morse function on a simplicial complex describes how to construct a homotopy-equivalent $\mathrm{CW}$-complex with possibly fewer cells. We associate a Boolean function with a given simplicial complex and construct a discrete Morse function using its Fourier transform.

Methods from theoretical computer science by O'Donnell, Saks, Schramm, and Servedio, together with experimental data on complexes from Hachimori's library and on chessboard complexes, provide some evidence that the constructed discrete Morse functions are efficient.

\section{INTRODUCTION}

Discrete Morse theory was introduced in [Forman 98] as a method to reduce the size of simplicial complexes (while preserving homotopy type) by collapsing interior parts of them. It was rapidly included as a standard weapon in the combinatorial topology arsenal and is frequently used. There are two equivalent ways to describe the collapsing process: by defining a partial matching on the face poset or by defining a real-valued map on the abstract simplicial complex. Such real-valued maps, satisfying some conditions to be stated later, are called discrete Morse functions. For example, the map whose value is the dimension of the cell is a discrete Morse function, but the associated collapsing does nothing.

A decision tree on a simplicial complex gives rise to a discrete Morse function [Forman 00], and for many complexes this is efficient [Jonsson 05]. Informally, a decision tree for a simplicial complex $\Delta$ with vertex set $V$ determines whether a subset $\sigma$ of $V$ is in $\Delta$ by asking questions of the type "Is $v$ in $\sigma$ ?" Sometimes it is not necessary to ask about all of $V$ to determine whether a $\sigma$ is in $\Delta$, and much research has been done on how to construct general decision trees that ask few questions. A good guideline is that the fewer questions in a decision tree, the better the associated discrete Morse function will be in the sense that the homotopy-equivalent CW-complex is small.

Decision trees for Boolean functions were studied in [Schramm et al. 05], and in the language of simplicial

(C) A K Peters, Ltd. 1058-6458/2009 \$0.50 per page Experimental Mathematics 18:1, page 45 
complexes, the authors proved that

$$
K_{\Delta} \leq \sum_{v \in V} \delta(T ; v) \widehat{\Delta}(\{v\}),
$$

where $K_{\Delta}$ is a constant depending only on $\Delta$. The probability of the question "Is $v$ in $\sigma$ ?" by the decision tree $T$ is $\delta(T ; v)$, and $\widehat{\Delta}(\{v\})$ is the Fourier transform of $\Delta$ at $\{v\}$. Exact definitions will be provided later. The important point is that if we want to minimize

$$
\sum_{v \in V} \delta(T ; v)
$$

we should try to get the $\delta(T ; v)$ for which $\widehat{\Delta}(v)$ is large to be comparably large. The probability of the question "Is $v$ in $\sigma$ ?" is measured by $\delta(T ; v)$, so the easy way to push up $\delta(T ; v)$ is simply to ask the question.

There are more elaborate ways to control $\sum_{v \in V} \delta(T ; v)$, as in [O'Donnell and Servedio 06, Servedio 04], but these methods typically focus on the running time of algorithms. For us the primary concern will be to construct discrete Morse functions from discrete Fourier transforms with as few critical cells as possible following a procedure that is topologically understandable.

\section{DISCRETE MORSE THEORY}

First let us fix some topological notation with a combinatorial flavor. An abstract simplicial complex with vertex set $V$ is a subset $\Delta$ of $2^{V}$ with the property

$$
\sigma \subseteq \tau \in \Delta \Rightarrow \sigma \in \Delta
$$

We deliberately allow $\varnothing \in \Delta$. The dimension of a cell $\sigma \in \Delta$ is $\# \sigma-1$, but we will use $\# \sigma$ more than $\operatorname{dim} \sigma$. The vertex set is from now on finite, and usually it is $[n]=\{1,2, \ldots, n\}$ or a subset of it. Sometimes we denote the vertices of $\Delta$ by $\Delta^{0}$.

Definition 2.1. A discrete Morse function on $\Delta$ is a function

$$
f: \Delta \rightarrow \mathbb{R}
$$

satisfying for all $\sigma \in \Delta$,

$$
\#\{\sigma \subset \tau \in \Delta \mid \# \tau=\# \sigma+1, f(\tau) \leq f(\sigma)\} \leq 1
$$

and

$$
\#\{\sigma \supset \tau \in \Delta \mid \# \tau=\# \sigma-1, f(\tau) \geq f(\sigma)\} \leq 1
$$

Modulo the empty set and the restriction from CWcomplexes to simplicial complexes, Definition 2.1 is the original one from [Forman 98]. There is an alternative definition in [Chari 00] that is more frequently used today, but it does not suit our setting as well. The state of the art of discrete Morse theory is surveyed in the book [Jonsson 08].

CW-complexes are more general structures than simplicial complexes. Often a topological space can be described in terms of fewer cells by a CW-complex than a simplicial complex, since the maps that glue cells together can be nontrivial; see [Lundell and Weingram 69] for definitions.

Definition 2.2. If $f$ is a discrete Morse function on $\Delta$, then the critical cells are all $\sigma \in \Delta \backslash\{\varnothing\}$ satisfying

$$
\#\{\sigma \subset \tau \in \Delta \backslash\{\varnothing\} \mid \# \tau=\# \sigma+1, f(\tau) \leq f(\sigma)\}=0
$$

and

$$
\#\{\sigma \supset \tau \in \Delta \backslash\{\varnothing\} \mid \# \tau=\# \sigma-1, f(\tau) \geq f(\sigma)\}=0
$$

As with an ordinary Morse function, the composition of a discrete Morse function and a continuous strictly increasing map $\mathbb{R} \rightarrow \mathbb{R}$ will not alter its behavior. In our setting everything is also finite and discrete, so we can always assume that a discrete Morse function $f$ is within any nontrivial interval on $\mathbb{R}$.

Theorem 2.3. (The main theorem of discrete Morse theory.) [Forman 98, 7.3 and 8.2] If $\mathcal{C}$ are the critical cells of a discrete Morse function, then there is a $C W$-complex with $\mathcal{C}$ as cells that is homotopy equivalent to $\Delta$.

The gluing maps of the CW-complex obtained in Theorem 2.3 are easily described, but we refer to [Forman 98] and [Jonsson 08] for their description. The deletion of a vertex $v$ in a simplicial complex $\Delta$ is

$$
\operatorname{dl}_{\Delta}(v)=\{\sigma \in \Delta \mid v \notin \sigma\},
$$

and the link of $v$ is

$$
\operatorname{lk}_{\Delta}(v)=\left\{\sigma \in \operatorname{dl}_{\Delta}(v) \mid \sigma \cup\{v\} \in \Delta\right\} .
$$

The vertex set of both $\mathrm{dl}_{\Delta}(v)$ and $\operatorname{lk}_{\Delta}(v)$ is $V \backslash\{v\}$ if $V$ is the vertex set of $\Delta$. Note that $\# \Delta=\# \mathrm{lk}_{\Delta}(v)+\# \mathrm{dl}_{\Delta}(v)$. The main technical detail of [Forman 00, Jonsson 05] is that discrete Morse functions for links and deletions can be merged.

Lemma 2.4. [Forman 00, Theorem 2.1] If $f_{\mathrm{dl}}: \mathrm{dl}_{\Delta}(v) \rightarrow$ $\left[0, \# \mathrm{dl}_{\Delta}(v)-1\right]$ and $f_{\mathrm{lk}}: \operatorname{lk}_{\Delta}(v) \rightarrow\left[0, \# \operatorname{lk}_{\Delta}(v)-1\right]$ are 
discrete Morse functions on the deletion and link of $\Delta$, then $f: \Delta \rightarrow[0, \# \Delta-1]$ defined by

$$
f(\sigma)= \begin{cases}f_{\mathrm{lk}}(\sigma \backslash\{v\})+\# \mathrm{dl}_{\Delta}(v) & \text { if } v \in \sigma \\ f_{\mathrm{dl}}(\sigma) & \text { if } v \notin \sigma\end{cases}
$$

is a discrete Morse function on $\Delta$.

\section{FOURIER TRANSFORMS}

In this section we introduce the concept of Fourier transforming simplicial complexes and show that the vertices of a simplicial complex with the maximal Fourier transform are the same as those with the minimal link size. For an introduction to Fourier analysis on finite abelian groups, and in particular on $\mathbb{Z}_{2}^{n}$, see [Terras 99]. Any function $\varphi:\{-1,1\}^{n} \rightarrow \mathbb{R}$ can be expanded as

$$
\varphi(x)=\sum_{\sigma \subseteq\{1,2, \ldots, n\}} \widehat{\varphi}(\sigma) \chi_{\sigma}(x),
$$

where $\chi_{\sigma}(x)=\prod_{i \in \sigma} x_{i}$ and

$$
\widehat{\varphi}(\sigma)=\mathbb{E}\left[\varphi(x) \chi_{\sigma}(x)\right]
$$

for the uniform probability distribution on $\{-1,1\}^{n}$. The influence of a coordinate as defined in [Ben-Or and Linial $90]$ is

$$
\operatorname{Inf}_{i}(\varphi)=\sum_{\sigma \subseteq\{1,2, \ldots, n\} \backslash\{i\}} \widehat{\varphi}(\sigma \cup\{i\})^{2},
$$

and for monotone $\varphi$ this equals $\widehat{\varphi}(\{i\})$ [Kahn et al. 88].

Definition 3.1. Let $\Delta$ be a simplicial complex with vertex set $[n]$. Let the monotone function $\varphi:\{-1,1\}^{n} \rightarrow$ $\{-1,1\}$ be given by $\varphi(x)=-1$ if $\left\{1 \leq i \leq n \mid x_{i}=1\right\} \in$ $\Delta$ and $\varphi(x)=1$ otherwise. The Fourier transform of $\Delta$ is the function $\widehat{\Delta}: 2^{[n]} \rightarrow \mathbb{R}$ defined by $\widehat{\Delta}(\sigma)=\widehat{\varphi}(\sigma)$.

In the introduction it was outlined that we want to ask questions about vertices with high $\widehat{\Delta}(\{v\})$.

Lemma 3.2. Let $\Delta$ be a simplicial complex with vertex set $[n]$. The elements $v$ of $[n]$ with the maximal $\widehat{\Delta}(\{v\})$ are the same as those with the minimal $\# \operatorname{lk}_{\Delta}(v)$.

Proof: Let $\varphi$ be the function defined by $f(x)=-1$ if $\left\{1 \leq i \leq n \mid x_{i}=1\right\} \in \Delta$ and $\varphi(x)=1$ otherwise. Using that $\varphi$ is monotone, we get that

$$
\begin{aligned}
\widehat{\Delta}(\{v\})= & \widehat{\varphi}(\{v\}]) \\
= & \mathbb{E}\left[\varphi(x) \chi_{\{v\}}(x)\right] \\
= & 2^{-n}(-\#\{\sigma \subseteq[n] \mid v \notin \sigma, \sigma \notin \Delta\} \\
& +\#\{\sigma \subseteq[n] \mid v \notin \sigma, \sigma \in \Delta\} \\
& +\#\{\sigma \subseteq[n] \mid v \in \sigma, \sigma \notin \Delta\} \\
& -\#\{\sigma \subseteq[n] \mid v \in \sigma, \sigma \in \Delta\}) \\
= & 2^{-n}\left(-2^{n-1}\right. \\
& +\#\{\sigma \subseteq[n] \mid v \notin \sigma, \sigma \in \Delta\} \\
& +\#\{\sigma \subseteq[n] \mid v \notin \sigma, \sigma \in \Delta\} \\
& \left.+2^{n-1}-\#\{\sigma \subseteq[n] \mid v \in \sigma, \sigma \in \Delta\}\right) \\
& -\#\{\sigma \subseteq[n] \mid v \in \sigma, \sigma \in \Delta\}) \\
= & 2^{1-n}(\#\{\sigma \subseteq[n] \mid v \notin \sigma, \sigma \in \Delta\} \\
& -\#\{\sigma \subseteq[n] \mid v \in \sigma, \sigma \in \Delta\}) \\
= & 2^{1-n}(\# \Delta-2 \#\{\sigma \in \Delta \mid v \in \sigma\}) \\
= & 2^{1-n}(\# \Delta-2 \#\{\sigma \in \Delta \mid v \notin \sigma, \sigma \cup\{v\} \in \Delta\}) \\
= & 2^{1-n}(\# \Delta-2 \# l \mathrm{k} \Delta(v)),
\end{aligned}
$$

which completes the proof.

Another piece of information we can get from the size of links is that if $2 \# \operatorname{lk}_{\Delta}(v)=\# \Delta$, then $\Delta$ is a cone with apex $v$. Thus the intuition that apices have zero Fourier transform is correct.

\section{CONSTRUCTING DISCRETE MORSE FUNCTIONS}

The easiest complexes on which to construct discrete Morse functions are those for cones, and we need the following description of a uniform way to do that.

Definition 4.1. Let $\Delta$ be a finite simplicial cone with apex $v$ and assume that its vertices are totally ordered. Order the cells of $\mathrm{dl}_{\Delta}(v)$ increasingly by dimension and then order the cells of the same dimension lexicographically with respect to the total order on the vertices: $\sigma_{0}, \sigma_{1}, \ldots, \sigma_{\# \Delta / 2-1}$. The standard discrete Morse function for the cone is defined by $f\left(\sigma_{i} \cup\{v\}\right)=i$ and $f\left(\sigma_{i}\right)=i+\# \Delta / 2$ for $0 \leq i<\# \Delta / 2$.

The reader is invited to check that the standard discrete Morse function for a cone is a discrete Morse function. As an example, let $\Delta$ be the complex on $\{1,2,3,4,5\}$ with facets 1245 and 234 , and the vertices given in the standard order. Then the discrete Morse function for apex 2 is given by 


\begin{tabular}{c|cccccccccc}
$\sigma$ & 2 & 12 & 23 & 24 & 25 & 124 & 125 & 234 & 245 & 1245 \\
\hline$f(\sigma)$ & 0 & 1 & 2 & 3 & 4 & 5 & 6 & 7 & 8 & 9
\end{tabular}

\begin{tabular}{c|cccccccccc}
$\sigma$ & $\varnothing$ & 1 & 3 & 4 & 5 & 14 & 15 & 34 & 45 & 145 \\
\hline$f(\sigma)$ & 10 & 11 & 12 & 13 & 14 & 15 & 16 & 17 & 18 & 19
\end{tabular}

A decision tree to determine whether a subset $\sigma$ of $\Delta^{0}$ is in $\Delta$ is an algorithm that asks questions of the type, "Is $v$ in $\sigma$ ?" for $v \in \Delta^{0}$. The algorithm is allowed to adapt the questions from the answers it has obtained. One common goal is the construction of decision trees that do not have to ask about all $v \in \Delta^{0}$ to decide whether a $\sigma$ is in $\Delta$ for any $\sigma$.

Decision trees for Boolean functions were studied in [Schramm et al. 05]. The authors proved that

$$
K_{\Delta} \leq \sum_{v \in V} \delta(T ; v) \widehat{\Delta}(\{v\}),
$$

where $\delta(T ; v)$ is the probability of the question, "Is $v$ in $\sigma$ ?" by the decision tree $T$. As noted in [Håstad 07], inequality (4-1) with its application to discrete Morse functions makes sense only if we have a nontrivial description of $K_{\Delta}$. Indeed, we have

$$
K_{\Delta}=\mathbb{V}[f]=2^{2-2 n}\left(2^{n}-\# \Delta\right) \# \Delta,
$$

where $\mathbb{V}[f]$ is the variance of the Boolean function associated with $\Delta$ in Definition 3.1.

The critical cells of a discrete Morse function derived from a decision tree as in [Forman 00] are exactly those cells $\sigma$ for which the question "Is $v$ in $\sigma$ ?" has been asked for all $v \in \Delta^{0}$. So if we want few critical cells, $\sum_{v \in \Delta^{0}} \delta(T ; v)$ should be kept small. But in inequality $(4-1)$, the constants $\widehat{\Delta}(\{v\})$ and $K_{\Delta}$ are nonnegative and do not depend on the decision tree, which puts a limit on how small $\sum_{v \in \Delta^{0}} \delta(T ; v)$ can be made.

If a decision tree starts off asking questions, and the right-hand side of $(4-1)$ is not over $K_{\Delta}$ when it has asked about almost all vertices of $\Delta$, then it will ask about the last vertex, and create a critical cell in the context of discrete Morse functions. Our goal is to avoid critical cells, so one natural strategy is to push up the righthand side of (4-1) as early as possible. This is most efficiently done by asking about the element with the highest Fourier transform.

In the definition of Morse-Fourier functions, we need a total order on the vertex set of the simplex to obtain a well-defined function, but one could define a class of functions.
Definition 4.2. Given a finite nonempty simplicial complex $\Delta$ with a total order on its vertices, the MorseFourier function $f: \Delta \rightarrow\{0,1, \ldots, \# \Delta-1\}$ is the bijection defined by

(i) if $\Delta=\{\varnothing\}$ then $f(\varnothing)=0$;

(ii) if $\Delta$ is a cone and $v$ is the first apex of a cone in the total order, then $f$ is the standard discrete Morse function for the cone $\Delta$ with apex $v$;

(iii) otherwise, let $v$ be the first vertex in the total order for which $\widehat{\Delta}(\{v\})=\max \left\{\widehat{\Delta}(\{u\}) \mid u \in \Delta^{0}\right\}$.

If $f_{\mathrm{dl}}: \mathrm{dl}_{\Delta}(v) \rightarrow\left[0, \# \mathrm{dl}_{\Delta}(v)-1\right]$ and $f_{\mathrm{lk}}: \mathrm{lk}_{\Delta}(v) \rightarrow$ $\left[0, \# \mathrm{lk}_{\Delta}(v)-1\right]$ are the Morse-Fourier functions for the deletion and link of $\Delta$, then

$$
f(\sigma)= \begin{cases}f_{\mathrm{lk}}(\sigma \backslash\{v\})+\# \mathrm{dl}_{\Delta}(v) & \text { if } v \in \sigma \\ f_{\mathrm{dl}}(\sigma) & \text { if } v \notin \sigma .\end{cases}
$$

After proving that Morse-Fourier functions are discrete Morse functions and giving an alternative definition, we will give a detailed example. The definition of the Morse-Fourier function is combinatorial, and the topological interpretation is not clear. There are, however, previous examples of interaction between Fourier transforms and topology. Arrow's impossibility theorem [Arrow 50] was proved in [Kalai 02] using Fourier methods and put into the context of hyperplane arrangements in [Terao 07].

Theorem 4.3. Let $\Delta$ be a finite nonempty simplicial complex with a total order on its vertices. Then its MorseFourier function as defined in Definition 4.2 is a discrete Morse function.

Proof: The proof is by induction over $\# \Delta^{0}$. If $\# \Delta^{0}=0$ then $\Delta=\{\varnothing\}$ and (i) defines a discrete Morse function.

Now assume that $\# \Delta^{0}>0$. If $\Delta$ is a cone, then $f$ is the standard discrete Morse function for a cone, and hence a discrete Morse function. Otherwise, $\Delta$ is split into the deletion and link of a vertex. By induction we know that the Morse-Fourier functions on the deletion and link are discrete Morse functions, since $\mathrm{dl}_{\Delta}(v)^{0}, \mathrm{lk}_{\Delta}(v)^{0} \subsetneq \Delta^{0}$. And by Lemma 2.4, their merged discrete Morse function is a discrete Morse function.

Alternatively, Morse-Fourier functions can be defined in a more enumerative way. 
Definition 4.4. Given a finite nonempty simplicial complex $\Delta$ with a total order on its vertices, the MorseFourier function $f: \Delta \rightarrow\{0,1, \ldots, \# \Delta-1\}$ is the bijection determined by first calculating $l(v)=\# \operatorname{lk}(v)$ for $v \in \Delta^{0}$ and then

(i) if $\Delta=\{\varnothing\}$ then $f(\varnothing)=0$;

(ii) if $2 l(u)=\# \Delta$ for any $u \in \Delta^{0}$, then let $v$ be the first such in the total order, and let $f$ be the standard discrete Morse function for the cone $\Delta$ with apex $v$;

(iii) otherwise, let $v$ be the first vertex in the total order for which $l(v)=\min \left\{l(u) \mid u \in \Delta^{0}\right\}$.

If $f_{\mathrm{dl}}: \mathrm{dl}_{\Delta}(v) \rightarrow\left[0, \# \mathrm{dl}_{\Delta}(v)-1\right]$ and $f_{\mathrm{lk}}: \mathrm{lk}_{\Delta}(v) \rightarrow$ $\left[0, \# \mathrm{lk}_{\Delta}(v)-1\right]$ are the Morse-Fourier functions for the deletion and link of $\Delta$, then

$$
f(\sigma)= \begin{cases}f_{\mathrm{lk}}(\sigma \backslash\{v\})+\# \mathrm{dl}_{\Delta}(v) & \text { if } v \in \sigma, \\ f_{\mathrm{dl}}(\sigma) & \text { if } v \notin \sigma .\end{cases}
$$

Theorem 4.5. Definitions 4.2 and 4.4 agree.

Proof: The differences between the definitions are in points (ii) and (iii). First point (ii). A vertex is the apex of a cone exactly when any cell can be extended with it. And if a vertex is in a cell in a simplicial complex, it can always be removed. Thus a vertex $v$ is the apex of the cone $\Delta$ exactly when $2 \# \operatorname{lk}(v)=\# \Delta$. Now point (iii). By Lemma 3.2, the vertices with maximal Fourier transform and minimal link size are the same.

\subsection{A Detailed Example}

The dunce hat [Zeeman 64] is an example of a contractible but not collapsible simplicial complex. A contractible complex has an odd number of critical cells, and a simplicial complex is collapsible exactly when there is a discrete Morse function with one critical cell. Hence we never get fewer than three critical cells from a discrete Morse function on a triangulation of the dunce hat. We will use a triangulation of the dunce hat from Hachimori's database [Hachimori 07]. It is illustrated in Figure 1 with the obvious identifications.

We construct the Morse-Fourier function of a complex $\Delta$ by pasting together Morse-Fourier functions from the link and deletion of a vertex with minimal link size, as in Definition 4.4. The convention of Figure 3 for links and deletions is described in Figure 2.

From now on, $\Delta$ is the dunce hat triangulated as in Figure 1, and its vertices are in the order

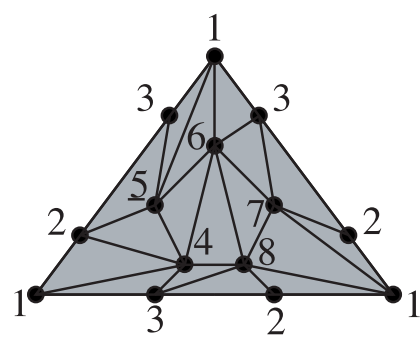

FIGURE 1. The dunce hat.

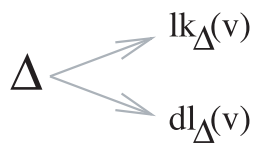

FIGURE 2. The convention of Figure 3 for links and deletions.

$1,2,3,4,5,6,7,8$. The decision tree that calculates $\Delta$ together with its Morse-Fourier function is drawn in Figure 3.

The link size calculations of $\Delta$ appear in Table 1 .

The size of the minimal link of $\Delta$ is 11 , and that is attained by vertices 5 and 7 . We split on vertex 5 . The reader is encouraged to have a look at Figure 3 at this point. All links drawn in Figure 3 are quite small, and the vertex with the smallest link size can be calculated directly. Note that the vertex on which we split is underlined in Figure 3.

Back to $\mathrm{dl}_{\Delta}(5)$. It is easy to prove that links and deletions commute, so to find $\operatorname{lk}_{\mathrm{dl}_{\Delta}(5)}(v)$, we look at $\mathrm{dl}_{\mathrm{lk}_{\Delta}(v)}(5)$ instead and use that $\mathrm{lk}_{\Delta}(v)$ was determined earlier.

According to Table 2, both vertices 4 and 6 have minimal link size, so we split at vertex 4 .

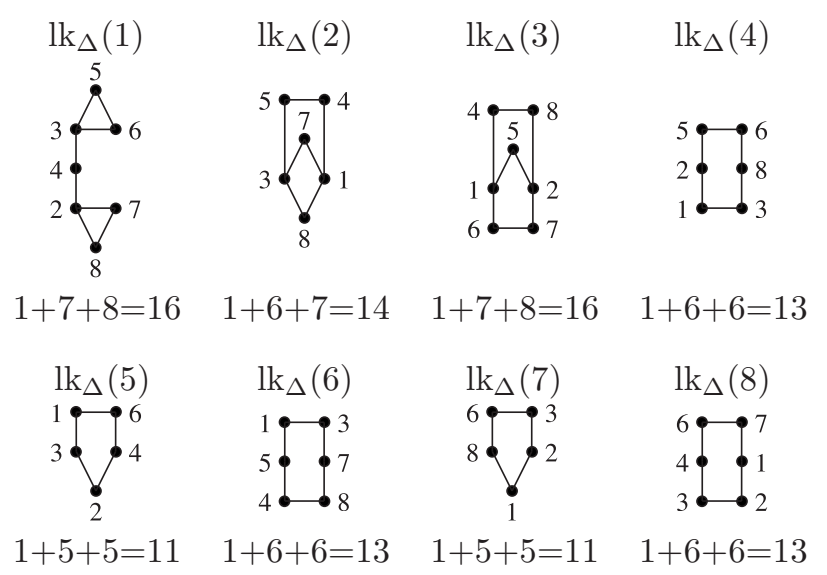

TABLE 1. Links of $\Delta$. 


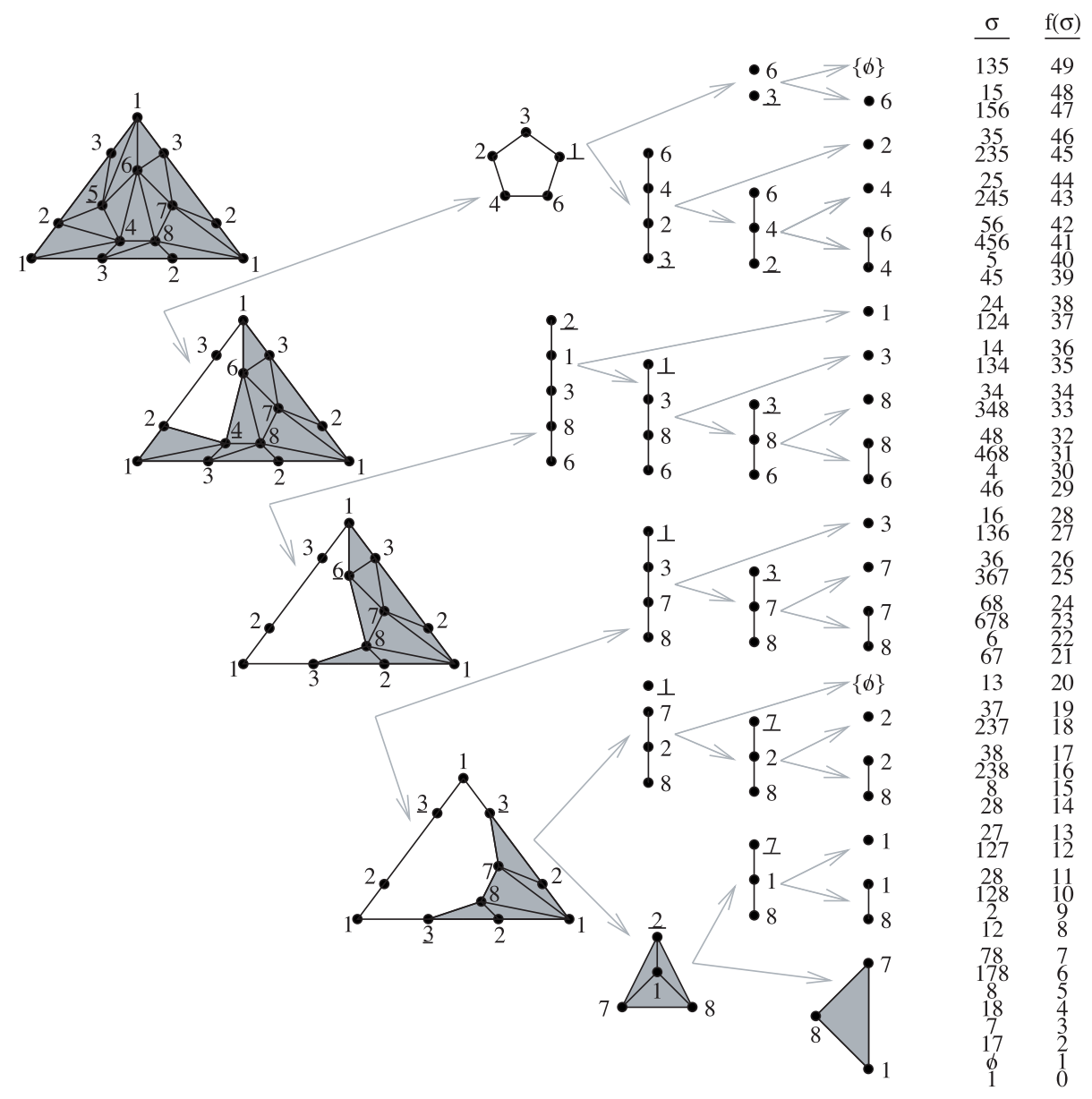

FIGURE 3. Calculation of the Morse-Fourier function $f$ for the dunce hat.

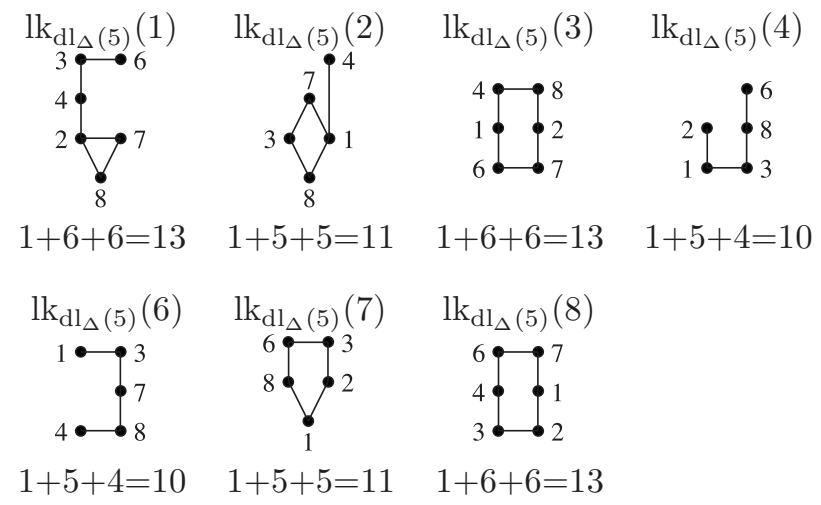

TABLE 2. Links of $\mathrm{dl}_{\Delta}(5)$.

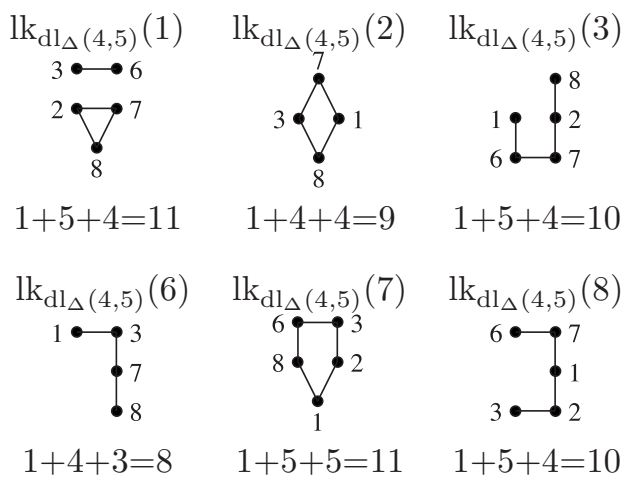

TABLE 3. The links of $\mathrm{dl}_{\Delta}(4,5)$.
From Table 3 we get that vertex 6 has the smallest link of $\mathrm{dl}_{\Delta}(4,5)$. By the same type of calculations, the smallest link of $\mathrm{dl}_{\Delta}(4,5,6)$ belongs to vertex 3 . The vertices 2,7 , and 8 have the smallest links in $\mathrm{dl}_{\Delta}(3,4,5,6)$, so we split at vertex 2 .

\subsection{Experimental Data}

In the first column of Table 4 are the names of some complexes we have tested for which to build Morse-Fourier functions. Then follow the number of faces, the number of critical cells, and a lower bound on the number of critical cells. 


\begin{tabular}{l|r|r|r} 
Complex & \#Faces & \#Critical & Lower Bound \\
\hline Bjorner & 32 & 2 & 2 \\
Dunce hat & 49 & 3 & 3 \\
Gruenbaum & 167 & 1 & 1 \\
Lockeberg & 216 & 4 & 2 \\
Mani-Walkup-C & 464 & 10 & 2 \\
Mani-Walkup-D & 392 & 8 & 2 \\
Nonextend & 39 & 3 & 1 \\
Non-PL sphere & 2680 & 16 & 6 \\
Poincare & 392 & 12 & 6 \\
Projective & 31 & 3 & 3 \\
Rudin & 215 & 5 & 1 \\
Ziegler & 119 & 1 & 1
\end{tabular}

TABLE 4. Simplicial complexes from Hachimori's library.

All of the complexes are from Hachimori's library [Hachimori 07]. Most of them have a combinatorially bad property. A good exercise is to download one or two of the small complexes and try to find discrete Morse functions for them by hand. Some of the discrete Morse functions from Fourier transforms listed above are optimal.

By a procedure described in [Forman 98] and later extended in [Hersh 05], some of the Fourier-Morse functions that are close to optimal can be made optimal, since they have only one gradient path between certain pairs of critical cells. The lower bound on the number of critical cells listed in Table 4 does not need to be exact, since optimal Morse functions are not known for all of the complexes. The nontrivial bounds are from [Hachimori 07] and [Joswig and Pfetsch 06], except for the bounds on Poincaré's homology sphere and its double one-point suspension (the non-PL sphere), which follows from the sizes of their homotopy groups [Stillwell 93].

Bjorner is Björner's example of a shellable but not extendably shellable complex. The Dunce hat has already been described. Gruenbaum is Grünbaum's example of a nonshellable triangulation of a 3-ball [Danaraj and Klee 78]. Lockeberg is a 4-dimensional polytope whose boundary complex is not vertex decomposable, found by Lockeberg [Klee and Kleinschmidt 87]. Mani-Walkup-C and Mani-Walkup-D are 3-spheres that are not vertex decomposable, constructed in [Mani and Walkup 80] as counterexamples to the $W_{w}$-path conjecture. Nonextend is a shellable but not extendably shellable complex found by Hachimori. Poincare is a triangulation from [Björner and Lutz 00] of the Poincaré homology sphere, and Non$P L$ sphere is its double one-point suspension, a non-PL sphere. An ordinary Projective plane is in the list. Rudin is Rudin's nonshellable triangulation of a 3-ball [Rudin
58]. Ziegler is a small nonshellable 3-ball from [Ziegler 98].

In [Joswig and Pfetsch 06], optimal Morse functions for some of the complexes in Table 4 were found. The authors also showed that the problem of finding optimal Morse functions is NP-complete and gave an integer programming formulation of it.

Several of the complexes described earlier were small enough to find an optimal discrete Morse function by brute force within days on a fast computer. Now we describe complexes with many thousands of cells for which the Morse-Fourier function can be calculated. The $n \times n$ chessboard complex has $[n]^{2}$ as vertex set, and its facets are $\{(1, \pi(1)),(2, \pi(2)), \ldots,(n, \pi(n))\}$, where $\pi$ is a permutation of $[n]$. There are many papers on the homology of chessboard complexes; see, for example, [Björner et al. 94, Shareshian and Wachs 07] and the survey chapter in [Jonsson 08]. Table 5 gives the number of critical cells of Morse-Fourier functions for different chessboard complexes.

Some enumerations of critical cells can be given combinatorial interpretations. For example, the numbers of $k$-dimensional critical cells of a $2 k \times 2 k$ chessboard complex are $16,142,274$ for $k=2,3,4$. The numbers of ways to partition $n$ labeled elements into pie slices of different sizes are $1,4,5,16,142,274,989,4288, \ldots$ according to sequence A032144 of [Sloan 07].

Conjecture 4.6. The number of $k$-dimensional critical cells of a Morse-Fourier function on a $2 k \times 2 k$ chessboard complex equals the number of ways to partition $k+3$ labeled elements into pie slices of different sizes.

We believe in a stronger statement.

Conjecture 4.7. There is an explicit description of the $C W$-complexes of critical cells from Morse-Fourier functions on chessboard complexes.

A simplicial complex is Morse-Fourier optimal if its Morse-Fourier function has the minimal number of critical cells for some ordering of the vertex set. Our experiments raise the natural question whether they are possible to characterize. From Table 4 we know that Grünbaum's nonshellable ball is Morse-Fourier optimal, so in particular we can get other collapsing sequences than from shellings.

\subsection{Maple Code}

Here we present an implementation in Maple 8 that is short but not fast. However, all of the previous exam- 


\begin{tabular}{|c|c|c|c|c|c|c|c|c|c|}
\hline Size \Dim & 0 & 1 & 2 & 3 & 4 & 5 & 6 & 7 & $\sum$ \\
\hline \multirow[t]{2}{*}{$3 \times 3$} & 9 & 18 & 6 & & & & & & 33 \\
\hline & 1 & 4 & 0 & & & & & & 5 \\
\hline \multirow[t]{2}{*}{$4 \times 4$} & 16 & 72 & 96 & 24 & & & & & 208 \\
\hline & 1 & 1 & 16 & 0 & & & & & 18 \\
\hline \multirow[t]{2}{*}{$5 \times 5$} & 25 & 200 & 600 & 600 & 120 & & & & 1545 \\
\hline & 1 & 0 & 13 & 69 & 0 & & & & 83 \\
\hline \multirow[t]{2}{*}{$6 \times 6$} & 36 & 450 & 2400 & 5400 & 4320 & 720 & & & 13326 \\
\hline & 1 & 0 & 1 & 142 & 326 & 0 & & & 470 \\
\hline \multirow[t]{2}{*}{$7 \times 7$} & 49 & 882 & 7350 & 29400 & 52920 & 35280 & 5040 & & 130921 \\
\hline & 1 & 0 & 0 & 17 & 1484 & 1671 & 0 & & 3173 \\
\hline \multirow[t]{2}{*}{$8 \times 8$} & 64 & 1568 & 18816 & 117600 & 376320 & 564480 & 322560 & 40320 & 1441728 \\
\hline & 1 & 0 & 0 & 1 & 274 & 15639 & 9157 & 0 & 25072 \\
\hline
\end{tabular}

TABLE 5. Morse-Fourier functions on chessboard complexes. For each size and dimension, the number of cells and critical cells are tabulated.

ples in Table 4 can be calculated in under a second on an ordinary desktop computer with this code. There is a small difference between this implementation and Definition 4.4 in how the discrete Morse functions for cones are constructed, but this does not change the number of critical cells per dimension. The algorithm works with the matching formulation by [Chari 00] of discrete Morse theory.

Start off with with (combinat): to include the standard combinatorial package. The input of $f()$ is the set of facets, and the output is the set of faces:

$f:=p$->map (op@powerset, $p$ ):

The input of $\mathrm{m}()$ is the set of faces, and the output is the discrete Morse matching from Fourier transforms:

$m:=p->$ if $\operatorname{nops}(p)=0$ then \{\} elif nops $(p)=1$ then $\{[o p(p)]\}$ else (V->if nops $(V)=1$ then $\{[o p(p)]\}$ else $(v->\operatorname{map}(1->\operatorname{map}(i->i$ union $\{v\}, I), m(\operatorname{map}(s->$ if $v$ in $s$ then $s$ minus $\{v\}$ end if,$p))$ )

union $m(\operatorname{map}(s->$ if not $(v$ in $s)$ then $s$ end if,p)) $((t->\min (o p(\operatorname{map}$ $(1->$ if $1[1]=\min (\operatorname{op}(\operatorname{map}(1->1[1], t)))$ then $1[2]$ end if,t)) ))

$(\operatorname{map}(v->[\operatorname{nops}(\operatorname{map}(s->$ if $v$ in $s$ then $s$ end if,p)),v],V)) ) end if) $(\operatorname{map}(o p, p))$ end if:

The input of $c()$ is a discrete Morse matching on a complex, and the output is the set of critical cells:

$c:=p->\operatorname{map}\left(s->\right.$ if $\operatorname{nops}(s)=1$ then op $(s)$ end if, $\operatorname{map}\left(s_{->}>\{\mathrm{op}(\mathrm{s})\}\right.$ minus $\{\{\}\}, p))$ :

This shows how to calculate the critical cells of a triangulation of the projective plane:

$(c @ m @ f)(\{\{1,3,4\},\{1,2,4\},\{1,2,6\},\{4,5,6\},\{3,4,6\}$,

$\{2,4,5\},\{2,3,6\},\{2,3,5\},\{1,5,6\},\{1,3,5\}\})$;

$\{\{1,2,4\},\{3,5\},\{6\}\}$

All of the code, with the complexes listed in the table, is available at http://www.math.kth.se/ alexe/ morseFourier/.

\section{ACKNOWLEDGMENTS}

The author thanks the referees for good suggestions, and Bruno Benedetti, Anton Dochtermann, Johan Håstad, and Jakob Jonsson for their comments and interesting discussions.

\section{REFERENCES}

[Arrow 50] K. Arrow. "A Difficulty in the Theory of Social Welfare." J. Political Economy 58 (1950), 328-346.

[Ben-Or and Linial 90] M. Ben-Or and N. Linial. "Collective Coin Flipping." In Randomness and Computation, pp. 91115. New York: Academic Press, 1990.

[Björner and Lutz 00] A. Björner and F. H. Lutz. "Simplicial Manifolds, Bistellar Flips and a 16-Vertex Triangulation of the Poincaré Homology 3-Sphere." Experiment. Math. 9:2 (2000), 275-289.

[Björner et al. 94] A. Björner, L. Lovász, S. T. Vrećica, and R. T. Živaljević. "Chessboard Complexes and Matching Complexes." J. London Math. Soc. (2) 49:1 (1994), 25-39.

[Chari 00] M. K. Chari. "On Discrete Morse Functions and Combinatorial Decompositions." Discrete Math. 217:1-3 (2000), 101-113.

[Danaraj and Klee 78] G. Danaraj and V. Klee. "Which Spheres Are Shellable?" Ann. Discrete Math. 2 (1978), $33-52$.

[Forman 98] R. Forman. "Morse Theory for Cell Complexes." Adv. Math. 134:1 (1998), 90-145.

[Forman 00] R. Forman. "Morse Theory and Evasiveness." Combinatorica 20:4 (2000), 489-504.

[Hachimori 07] M. Hachimori. "Simplicial Complex Library." Available online (http://infoshako.sk.tsukuba.ac. jp/ hachi/math/library/index_eng.html), 2007.

[Håstad 07] J. Håstad. Personal communication, 2007.

[Hersh 05] P. Hersh. "On Optimizing Discrete Morse Functions." Adv. in Appl. Math. 35:3 (2005), 294-322. 
[Jonsson 05] J. Jonsson. "Optimal Decision Trees on Simplicial Complexes." Electron. J. Combin. 12 (2005), Research Paper 3 (electronic).

[Jonsson 08] J. Jonsson. Simplicial Complexes of Graphs, Lecture Notes in Mathematics 1928. Berlin: SpringerVerlag, 2008.

[Joswig and Pfetsch 06] M. Joswig and M. E. Pfetsch. "Computing Optimal Morse Matchings." SIAM J. Discrete Math. 20:1 (2006), 11-25 (electronic).

[Kahn et al. 88] J. Kahn, G. Kalai, and N. Linial. "The Influence of Variables on Boolean Functions." In Proc. 29th Ann. Symp. Foundations of Comp. Sci., pp. 66-80. Washington, DC: Computer Society Press, 1988.

[Kalai 02] G. Kalai. "A Fourier-Theoretic Perspective on the Condorcet Paradox and Arrow's Theorem." Adv. in Appl. Math. 29:3 (2002), 412-426.

[Klee and Kleinschmidt 87] V. Klee and P. Kleinschmidt. "The $d$-Step Conjecture and Its Relatives." Math. Oper. Res. 12:4 (1987), 718-755.

[Lundell and Weingram 69] A. Lundell and S. Weingram. The Topology of $C W$ Complexes. New York: Van Nostrand Reinhold Company, 1969.

[Mani and Walkup 80] P. Mani and D. W. Walkup. "A 3-Sphere Counterexample to the $W_{v}$-Path Conjecture." Math. Oper. Res. 5:4 (1980), 595-598.

[O'Donnell and Servedio 06] R. O'Donnell and R. A. Servedio. "Learning Monotone Decision Trees in Polynomial Time." In CCC '06: Proceedings of the 21st Annual IEEE Conference on Computational Complexity, pp. 213-225. Washington, DC: IEEE Computer Society, 2006.
[Rudin 58] M. E. Rudin. "An Unshellable Triangulation of a Tetrahedron." Bull. Amer. Math. Soc. 64 (1958), 90-91.

[Schramm et al. 05] O. Schramm, R. O'Donnell, M. Saks, and R. A. Servedio. "Every Decision Tree Has an Influential Variable." In FOCS '05: Proceedings of the 46th Annual IEEE Symposium on Foundations of Computer Science, pp. 31-39. Washington, DC: IEEE Computer Society, 2005.

[Servedio 04] R. A. Servedio. "On Learning Monotone DNF under Product Distributions." Inform. and Comput. 193:1 (2004), 57-74.

[Shareshian and Wachs 07] J. Shareshian and M. L. Wachs. "Torsion in the Matching Complex and Chessboard Complex." Adv. Math. 212:2 (2007), 525-570.

[Sloan 07] N. J. A. Sloan. "The On-Line Encyclopedia of Integer Sequences." Available online (www.research.att. com/ njas/sequences/), 2007.

[Stillwell 93] J. Stillwell. Classical Topology and Combinatorial Group Theory, Graduate Texts in Mathematics 72, second edition. New York: Springer-Verlag, 1993.

[Terao 07] H. Terao. "Chambers of Arrangements of Hyperplanes and Arrow's Impossibility Theorem." Adv. Math. 214:1 (2007), 366-378.

[Terras 99] A. Terras. Fourier Analysis on Finite Groups and Applications, London Mathematical Society Student Texts 43. Cambridge: Cambridge University Press, 1999.

[Zeeman 64] E. C. Zeeman. "On the Dunce Hat." Topology 2 (1964), 341-358.

[Ziegler 98] G. M. Ziegler. "Shelling Polyhedral 3-Balls and 4-Polytopes." Discrete Comput. Geom. 19:2 (1998), 159174.

Alexander Engström, Department of Mathematics, Royal Institute of Technology, S-100 44 Stockholm, Sweden

(alexe@math.kth.se)

Received July 27, 2007; accepted in revised form August 20, 2008. 